\title{
Perspectives for the radiography of Mt. Vesuvius by cosmic ray muons
}

\author{
S. Buontempo ${ }^{1}$, L. D’Auria ${ }^{2}$, G. De Lellis ${ }^{1,3}$, G. Festa ${ }^{3}$, P. Gasparini ${ }^{3,4}$, G. Iacobucci ${ }^{1}$, A. Marotta ${ }^{1}$, \\ M. Martini ${ }^{2}$, G. Miele ${ }^{1,3}$, P. Migliozzi ${ }^{1}$, O. Pisanti ${ }^{1,3}$, P. Strolin $^{1,3}$, M. Vassallo ${ }^{4}$, and A. Zollo ${ }^{3}$ \\ ${ }^{1}$ Sezione di Napoli, Istituto Nazionale di Fisica Nucleare (INFN), Italy \\ ${ }^{2}$ Osservatorio Vesuviano, Istituto Nazionale di Geofisica (INGV), Napoli, Italy \\ ${ }^{3}$ Dip. di Scienze Fisiche, Università di Napoli Federico II, Italy \\ ${ }^{4}$ Consorzio Analisi e Monitoraggio Rischi Ambientali (AMRA), Napoli, Italy
}

(Received November 27, 2008; Revised April 14, 2009; Accepted May 26, 2009; Online published February 22, 2010)

\begin{abstract}
The measurements performed in Japan have shown that muon radiography is an "imaging technique" capable of providing information of the internal structure of volcanoes with a resolution and richness of details beyond the reach of conventional, non-imaging techniques. The measurements have been performed using electronic detectors or nuclear emulsions. The latter have shown excellent muon tracking capabilities and space resolution, but are lacking of the capability of electronic detectors to provide data in real time. In this paper, we examine the possibility of developing an electronic detector giving a resolution comparable to that of nuclear emulsions and with a larger area than used so far, in order to see deeper structures inside volcanoes in spite of the strong muon absorption in the rock. We specifically discuss the very challenging application of muon radiography to Mt. Vesuvius, driven by the strong social interest coming from the enormous potential danger which it represents. Applications to other volcanoes can be envisaged.
\end{abstract}

Key words: Volcanology, muons, cosmic rays, radiography.

\section{Introduction}

Muon radiography was first proposed to determine the thickness of snow layers on a mountain (George, 1955). The first application of this method was realised to search for unknown burial cavities in the Chephren's pyramid (Alvarez et al., 1970). The pioneering work done in Japan for the radiography of the edifice of volcanoes by using quasi-horizontal cosmic ray muons (Nagamine et al., 1995; Tanaka et al., 2003, 2005, 2007a-c, 2008; Tanaka and Yokoyama, 2008) opened new possibilities for the study of their internal structure. The spatial resolution that in principle can be reached in investigations of internal structures is of the order of tens of meters, which cannot be achieved with other techniques.

The present paper concerns the possibility to apply muon radiography to study the edifice of a volcano at very high risk, Mt. Vesuvius. Mt. Vesuvius is a cone, $1280 \mathrm{~m}$ high above sea level, which has grown within the ancient caldera of Mt. Somma (Figs. 1 and 2). Mt. Somma was formed about 19,000 years ago and its original height was estimated to be about $2000 \mathrm{~m}$. Only the northern ridge of Mt. Somma is actually left as a part of the ancient structure, whose collapse started during two large eruptions occurring about 18,000 and 16,000 years ago. The history of the volcano is characterized by dramatic Plinian and sub-Plinian eruptions, the most famous of which caused the destruction of the towns of Pompeii, Herculaneum and Stabiae in

Copyright (C) The Society of Geomagnetism and Earth, Planetary and Space Sciences (SGEPSS); The Seismological Society of Japan; The Volcanological Society of Japan; The Geodetic Society of Japan; The Japanese Society for Planetary Sciences; TERRAPUB.

doi:10.5047/eps.2009.05.005
AD 79, as reported by Pliny the Younger (Plinius, 104). In relatively recent times several eruptions occurred, the most recent in 1944. The eruptions altered dramatically the morphology of the mountain, as it can be deduced from the artistic representation shown in Fig. 3. An impressive picture of the evolution in the course of the years 1630-1944 can be found in http://www.ov.ingv.it/. At present, the monitoring system does not reveal signs of notable activity. The present apparent quiescence raises questions on the possible storing of a large amount of energy that could be delivered in a future eruption.

The population living at the base and along the slopes of Mt. Vesuvius in a "red" area, which is classified at the highest volcanic risk in Europe, reaches about 600,000 people. The knowledge of the inner structure of the volcano edifice and subsoil structure is of the greatest importance to build realistic scenarios of the future eruptive activity through simulations of the magma upraising mechanism and eruption.

Mt. Vesuvius is among the most studied volcanoes in the world. It was thoroughly investigated using geophysical methods. The Vesuvian Observatory was set up in 1841 . It is the oldest volcano observatory in the world. Its ancient building was constructed on the volcano slopes at about $600 \mathrm{~m}$ above sea level. The main research activities of the Vesuvian Observatory, which is now also in charge of monitoring Stromboli, were moved to the headquarters in Naples. The historically pioneering research infrastructure at Mt. Vesuvius is still operational by hosting monitoring instruments and a scientific museum.

The study Mt. Vesuvius by muon radiography represents a very challenging task, due both to the morphology of the 


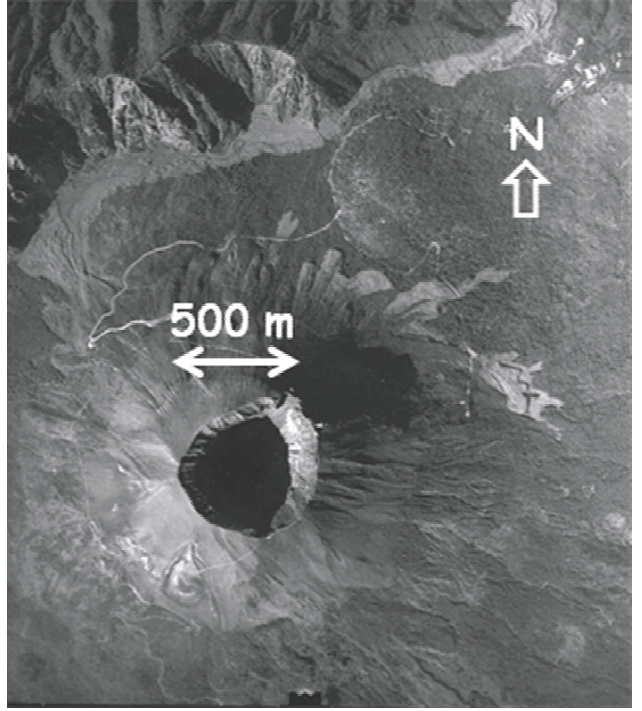

Fig. 1. Aerial view of Mt. Vesuvius; part of Mt. Somma is visible in the upper side.

mountain and to the aim of investigating deeper structures than in previous applications of muon radiography to volcanoes. The use of this technique to investigate the internal structures of volcano edifices at greater depths below their summit, where absorption strongly reduces the muon flux, requires detectors of larger area than previously built (of the order of $1 \mathrm{~m}^{2}$ ) though maintaining features of portability in a volcanic environment. Detectors of larger area reduce the time required for a radiography and, in applications at shallower depths, may aim to the (quasi) real time response required for monitoring purposes. Together with a large area, it is important to achieve a high angular resolution, of the order of $15 \mathrm{mrad}$. So far, such a resolution has been achieved only by using nuclear emulsions (Tanaka et al., 2007a-c, 2008; Tanaka and Yokoyama, 2008).

The realisation of a large-area, high-resolution detector for the study of Mt. Vesuvius, exploiting new technologies developed for Particle Physics, has a broader interest in view of its utilization for the study of other volcanoes. We thus intend to operate in the framework of a scientific network sharing our objectives.

This paper is associated with those presented by G. Miele and by M. Vassallo at this workshop.

\section{Volcanological Motivations}

Mt. Vesuvius is a stratovolcano located in Southern Italy within a graben (Campania Plain) formed in the PlioPleistocene. It is the southernmost and the youngest of a group of Pleistocene volcanoes, three of which (Mt. Epomeo in Ischia Island, Phlegrean Fields and Mt. Vesuvius) erupted in historical times. Mt. Vesuvius is located $15 \mathrm{~km}$ east of the city of Naples. The AD 79 eruption destroyed Pompeii, Herculaneum and Stabiae. It is the first catastrophic eruption described in remarkable details (Plinius, 104).

The chronology of Mt. Vesuvius eruptions shows that at least seven violent Plinian as well as numerous sub-Plinian eruptions occurred in historical times, many of which were separated by long periods of quiescence. The chronology suggests also that the longer is the quiescence period the more violent is the renewal of activity. The last period of persistent volcanism of Mt. Vesuvius started after the 1631 Plinian eruption and lasted till 1944. Since that time Mt. Vesuvius has been quiescent and actually only a moderate seismicity and fumaroles testify its activity.

The volcanic activity of Mt. Vesuvius is monitored by a multi-parametric observation system for the continuous recording of seismicity, ground-deformation and gasemission data that are transmitted to the operating centre of Vesuvian Observatory, located downtown in Naples.

The volcano was the object of accurate geophysical and volcanological studies. In particular, active/passive seismic tomography and wave reflection studies were recently performed with the aim of improving the knowledge of the deep geological structures beneath the volcano (Zollo et al., 1996, 2002; Auger et al., 2001). The structural model of the volcano is essential to predict realistic scenarios of the eruptive activity and it is the basis for the identification of possible precursors of future eruptions.

The resolution of seismic and more generally of geophysical techniques (seismological, electromagnetic, gravitational) is rather low, being able to define space variations of elastic properties with an accuracy of several hundreds of meters in the optimal acquisition conditions. This resolution does not allow characterizing small volcanic structures (conduits, high density anomalies, magma intrusions) that can have an important role in forecasting the time and magnitude of hazardous future eruptions.

With resolutions up to tens of meters in optimal detection

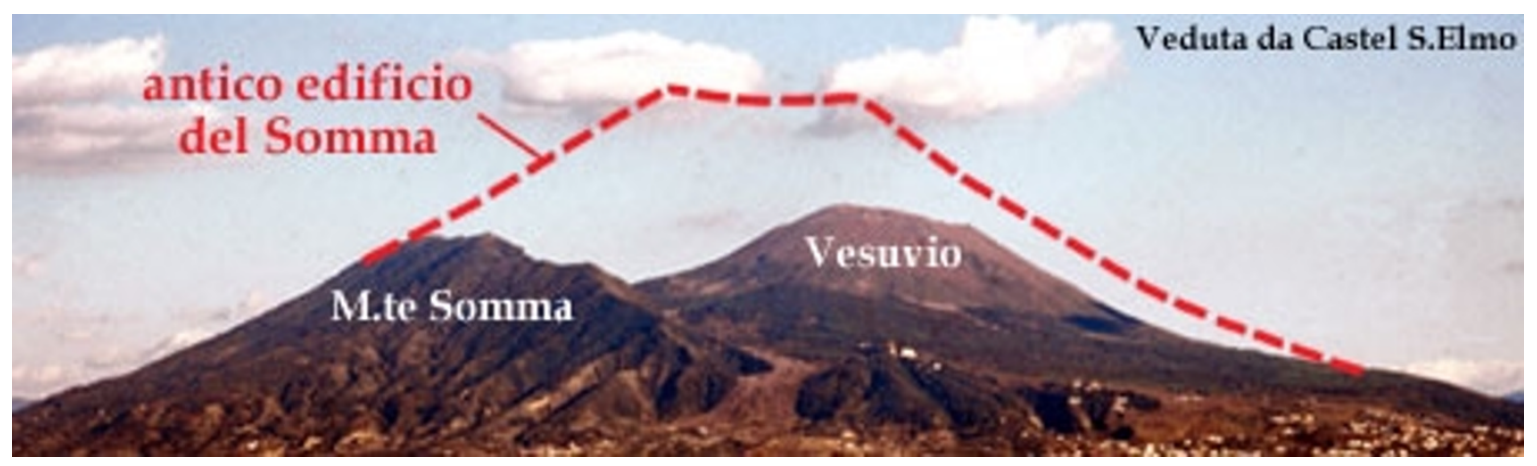

Fig. 2. Mt. Vesuvius in the caldera of Mt. Somma, as seen from Naples. 


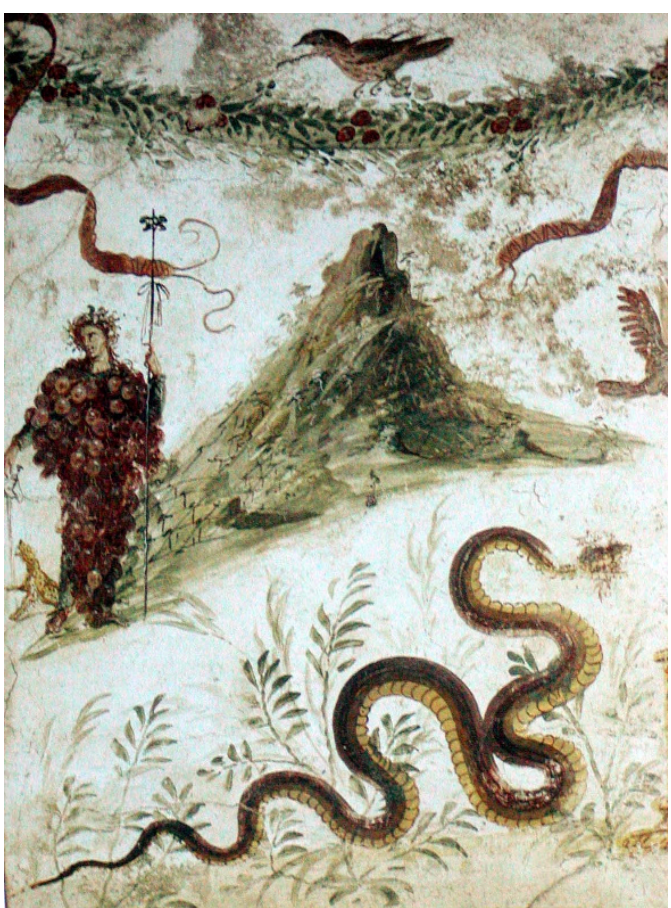

Fig. 3. Dionysus and Mt. Somma (presumably) before the AD 79 eruption (fresco from Casa del Centenario, Pompei; now at Naples Archaeological Museum).

conditions, muon radiography is expected to give us considerably better images of the top region of a volcano edifice than those obtained by conventional gravity methods. Muon radiography should thus allow to gather information on anomalies in the density distribution, such as those expected from dense lava conduits, low-density magma supply paths or the compression with depth of the overlying soil. The availability of accurate rock-density measurements from muon radiography should also allow inferring the chemical composition and thermal state of the volcano crater.

The combined use of muon radiography and seismological and gravity measurements is expected to provide a wide vision of the shallow/deep internal volcanic structure including also the topmost part of the volcano edifice that is important to monitor the state of the volcano and provide useful data to predict the evolution of an eruption.

The persistent eruptive activity and edifice morphology makes of "strombolian" volcanoes the ideal laboratory to exploit muon radiography techniques. The Stromboli volcano of the Aeolian Islands in southern Italy is considered one of the most active volcanoes in the world. Its persistent but moderate explosive activity is only interrupted by rare episodes of more vigorous activity accompanied by lava flows. This volcano is particularly suited for the application of muon radiography technique, since it is an open conduit volcano characterized by persistent conduit degassing and explosive dynamics. A detailed image of the volcanic edifice can help to map the conduit network path and monitor the eruption processes by surveying the magma emplacement at shallow depths close to the vents (Acocella et al., 2006; Chouet et al., 2008). Stromboli is a composite stratovolcano that steeply reaches $\sim 900 \mathrm{~m}$ above sea level, thus allowing to image a large portion of the volcano edifice by muon radiography.

The feasibility of periodically repeated cosmic-ray muon radiographies opens also new perspectives on the near-real time investigation of volume deformation of a volcanic edifice structure, induced by inner processes of mass redistribution. The occurrence of inflation episodes due to magma upraising or hydro-thermal fluid circulation can be the cause for significant volume and rock physical property changes which can produce density variations detectable by cosmic ray muon radiography. This would have great relevance for the monitoring of volcanic hazard and quantitative assessment of volcanic risk.

\section{Estimate of Muon Rates}

As an important premise we stress that reliable estimates of the muon flux are difficult to perform, because of their strong absorption in the rock to be traversed and of the uncertainties in simulating the interactions of muons at much higher energy than in current particle physics experiments. The estimate of the muon flux has thus to come from a Monte Carlo simulation tuned on real data.

For energies larger than $100 \mathrm{GeV}$, the cosmic-ray muon flux at the top of the atmosphere is described by the following expression (Adair and Kasha, 1976):

$$
\frac{d N}{d S d t d E d \Omega}=0.15 E^{-2.7}\left[\begin{array}{r}
\left.\frac{0.9}{1+\frac{1}{92} E \cos \theta}+\frac{0.1}{1+\frac{1}{540} E \cos \theta}\right] \\
\left(\mathrm{cm}^{2} \mathrm{~s} \mathrm{GeV} \mathrm{sr}\right)^{-1}
\end{array}\right.
$$

where $\theta$ denotes the zenith angle (measured from the vertical) and $E$ is the energy measured in GeV. The muon flux falls steeply with energy, with a power index -2.7 . This behaviour makes measurements requiring the traversal of large thicknesses of rock increasingly difficult. To set the energy scale of interest for muon radiography of volcanoes, let's consider that the ionisation energy loss of relativistic particles in matter is approximately $2.5 \mathrm{MeV} \mathrm{g}^{-1} \mathrm{~cm}^{-2}$ (The review of particle physics-Particle Data Group, Amsler et al., 2008). With a density of $2.2 \mathrm{~g} \mathrm{~cm}^{-3}$, it corresponds a loss of about $0.5 \mathrm{TeV} \mathrm{km}{ }^{-1}$. Accounting for high energy processes, the threshold for muons to traverse $1 \mathrm{~km}$ of rock is approximately $1.5 \mathrm{TeV}$.

By taking into account the energy loss in the atmosphere, the curvature of the earth and the possible decay of muons travelling towards the earth surface, one gets a new expression for the muon flux at the sea level as a function of the arrival direction. In Fig. 4 two parameterizations (Adair and Kasha, 1976; The review of particle physics-Particle Data Group, Amsler et al., 2008) for $\theta=70^{\circ}$ are shown. In the $\mathrm{TeV}$ energy region, which is relevant for our application, the two parameterizations are in agreement.

In order to obtain a preliminary estimate of the product (detector area) $\times$ (exposure time) needed to optimize the muon detector, we performed a simplified Monte Carlo calculation. We considered a simple geometrical model for the volcano and for the presence of an internal structure with diameter $\Phi$, as illustrated in Fig. 5. By taking into account 


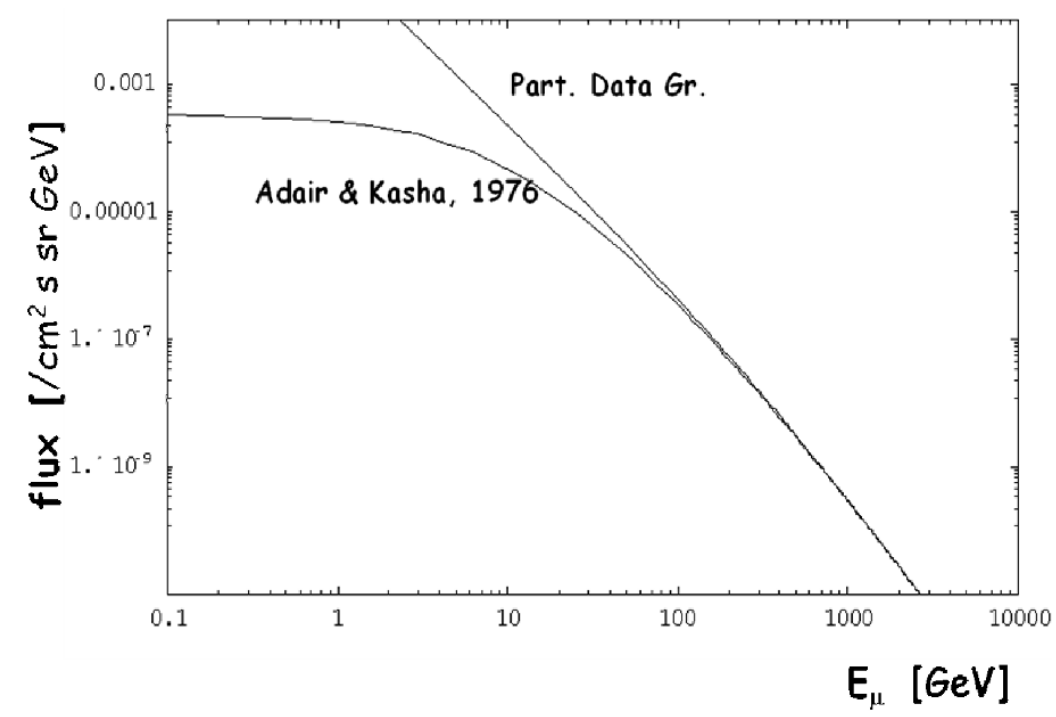

Fig. 4. The expected muon flux at the sea level for an arrival direction of $70^{\circ}$ with respect to the vertical.
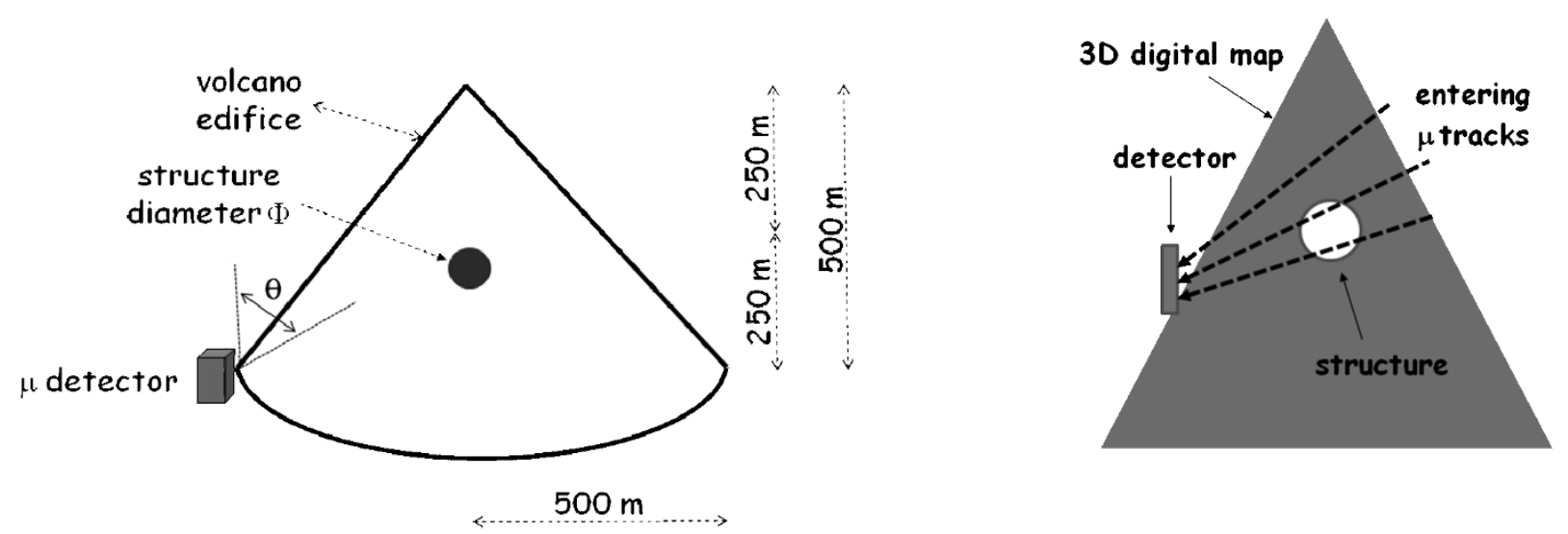

Fig. 5. Simplified model for the mountain-detector system, with an internal structure to be studied.

the muon energy loss in the rock and their survival probability (The review of particle physics-Particle Data Group, Amsler et al., 2008) we obtained the results shown in Table 1 , which reports the flux of muons with energy larger than $0.5 \mathrm{GeV}$ entering the detector and the (detector area) $\times(\mathrm{ex}-$ posure time) required to reveal, at the $3 \sigma$ significance level, an internal structure in two different cases: an empty cavity of $20 \mathrm{~m}$ diameter and a structure of $50 \mathrm{~m}$ diameter characterized by a $10 \%$ different density. The results of the model calculations indicate that detector areas of several square meters are required.

A full Monte Carlo simulation is needed for a better evaluation of the muon flux and of the multiple scattering in the rock. This full simulation is in progress. It is performed using the GEANT4 (Geometry ANd Tracking) toolkit for the simulation of the passage of particles through matter (http://www.geant4.org/geant4/package) and it features the 3D Digital Elevation Map (DEM) of Mt. Vesuvius, shown in Fig. 6, as geometrical input. It is based on a two steps technique, already developed for studying the matter effect for the neutrino detection (Miele et al., 2006) at the Pierre Auger observatory in South America (http://www.auger.org/) and the optimal configuration
Table 1. The product of detector area times measurement time required to see substructures, according to the simplified model of Fig. 5.

\begin{tabular}{|c|c|c|}
\hline$\Phi$ of structure & $\begin{array}{l}20 \\
\rho=0\end{array}$ & $\begin{array}{c}50 \\
\Delta \rho / \rho_{0}=0.1\end{array}$ \\
\hline $\begin{array}{l}\text { Rate N through structure }\left[m^{-2} \text { day }^{-1}\right] \\
\left(\rho_{0}=2.2 \mathrm{~g} \mathrm{~cm}^{-3}, E_{\mu}>0.5 \mathrm{GeV}\right)\end{array}$ & 2.5 & 16 \\
\hline$\Delta \mathrm{N} / \mathrm{N}$ by structure & 0.09 & 0.02 \\
\hline Events for $3 \sigma$ detection of structure & 1,150 & 20,000 \\
\hline Detector area $\times$ time & 15 & 40 \\
\hline
\end{tabular}

of the NEMO neutrino observatory in the Mediterranean Sea (http://nemoweb.lns.infn.it/project.htm). Indeed, the method is in essence the same for neutrinos or muons as incoming particles. As in the case of Fig. 5, we assumed as a starting point a given internal structure for the volcano including the presence of the substructures to be revealed. Then, as a first step, we generated a large number of cosmicray muons, which are tracked backwards from the detector to intersect the DEM and the substructures. For each track 


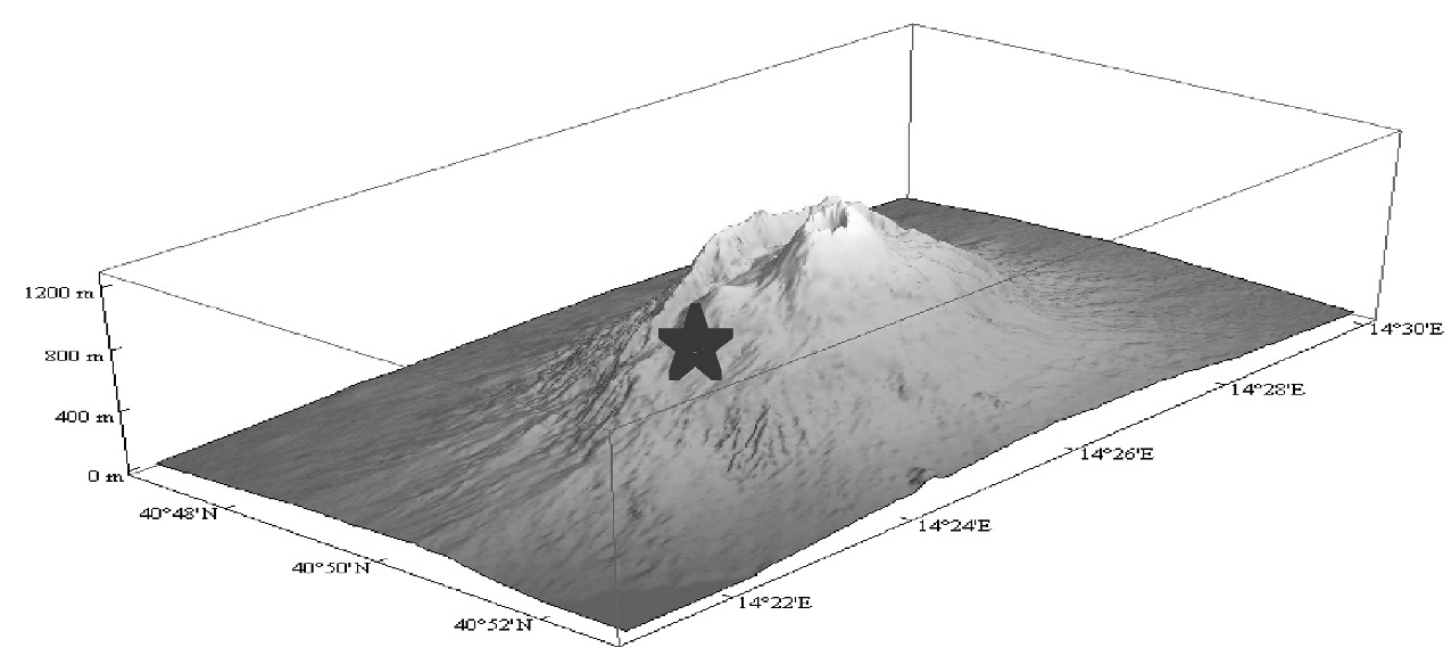

Fig. 6. The 3D Digital Elevation Map of the complex Mt. Vesuvius-Mt. Somma and the location ( $\star$ ) of the Vesuvian Observatory.

the layers of matter crossed and their densities are recorded, so that each track is characterised by a sequence of layers of matter ending at the detector. A huge set of muon tracks is quickly produced in this way. In a second step, the GEANT4 programme is used to simulate the interaction of the muons with the layers of matter they traverse.

At present, this technique is being applied by some of us (Gonzalez-Garcia et al., 2008) to the NEMO detector to perform the same study of the earth core-mantle boundary determination that was done by the IceCube neutrino observatory (http://icecube.wisc.edu/). To this purpose we have developed a new Monte Carlo code, which is obtained by combining the HERWIG hadron generator (Corcella et al., 2001) and GEANT4 in order to be able to treat neutrino interactions. The above Monte Carlo simulation technique will be used to estimate the muon transmission rate for different paths inside Mt. Vesuvius and thus obtain the measurement sensitivity for a given detector area and measurement time. Once this is achieved, we will be able to optimize the detector location taking into account the morphology of Mt. Vesuvius and practical considerations such as the ease of access and the availability of electrical power.

\section{The Muon Detector}

The success of the experiments conducted in Japan leads to envisage an increasing demand for muon radiography of volcanoes, with higher sensitivity. The spectrum of cosmic ray muons decreases according to the very steep power law $E^{-2.7}$, so that the flux of muons surviving the energy loss in the traversal of the volcano's edifice decreases very rapidly when deeper structures need to be investigated.

The active area of the muon detectors employed so far was at most $1 \mathrm{~m}^{2}$. This relatively small area limits the application to small volcanoes or to the summit (a few hundreds of meters) of larger volcanoes. As shown in the previous section, the extension of muon radiography to deeper structures requires an increase of the detector area by one order of magnitude. In addition, future detectors should aim at angular resolutions as small as $15 \mathrm{mrad}$, so far achieved only with nuclear emulsions. At $1 \mathrm{~km}$ distance this corresponds to a $15 \mathrm{~m}$ spatial resolution in the determination of internal structures. The deterioration of the spatial resolution due to the multiple scattering in the rock will have to be carefully estimated and is expected to have a comparable value. The detector will consist of a sequence of $(x-y)$ detector planes, to form what in Particle Physics is called a "telescope". A telescope is capable of measuring position and angle of particles, of which for muon radiography only the angle matters, as the detector is essentially pointlike with respect to the mountain.

In recent years, the need of particle-physics collider and neutrino experiments to cover very large detection areas (thousands of square meters) has driven a substantial progress in the development of detectors technologically more advanced, cheaper and simpler to handle. A new generation of detectors for muon radiography may profit of these developments. However, for application in a volcanic environment the detector must be characterized by portability, little need of maintenance as well as low electrical power consumption to allow the use of solar panels or other autonomous power supplies.

In the following, we briefly review those detector techniques that may be of interest for our purposes.

\subsection{Nuclear emulsions}

The very high spatial (hence angular) resolution of nuclear emulsions was evident in the beautiful results of recent muon radiographies of volcanoes (Tanaka et al., 2007a-c, 2008; Tanaka and Yokoyama, 2008), although not fully exploited because of the available statistics. Nuclear emulsions have undergone an impressive progress in their industrial production and in the analysis by high speed automated microscopes (as reported at the 3rd Int. Workshop on Nuclear Emulsion Technique, http://flab.phys.nagoyau.ac.jp/workshop/2008/index.html). They offer the advantage of needing no electricity supply. However, they do not allow real-time data acquisition and online transmission to laboratories for analysis.

\subsection{Resistive Plate Chambers}

In Resistive Plate Chambers (RPC) a gas mixture flows in a $2 \mathrm{~mm}$ gap, where an electric field is created by "resistive" 

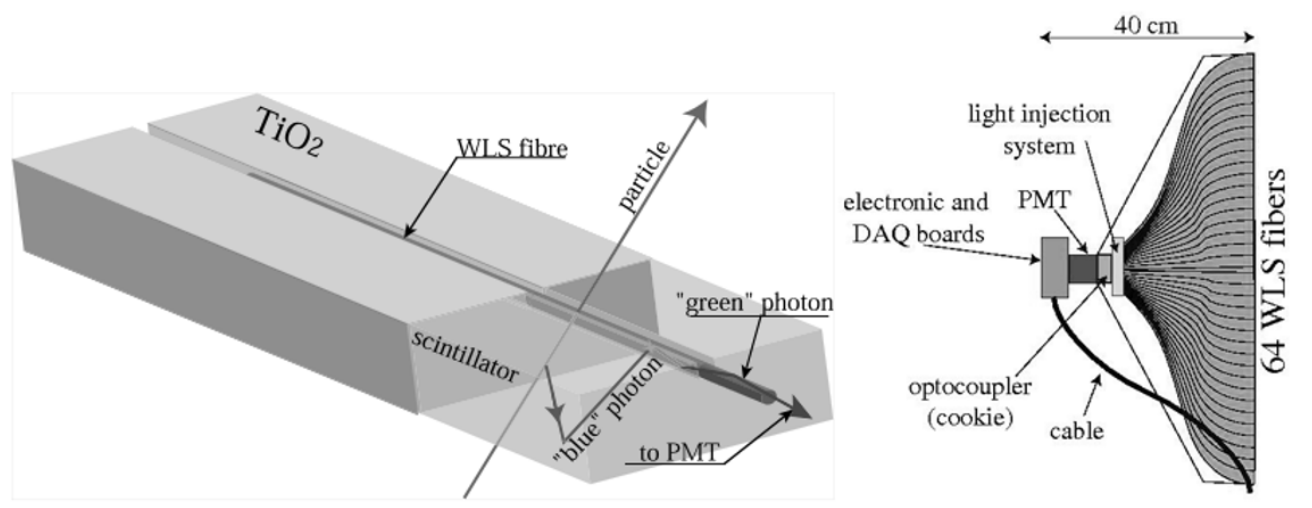

Fig. 7. The plastic scintillator strips (left figure) of the OPERA neutrino experiment and their readout system made by optical wavelength shifting fibres and multianode PMT (right figure).

electrodes which are transparent to the fast electrical signal produced by the passage of a charged particle. The signal can thus be readout by induction on metallic strips situated outside the chamber. A single chamber allows the readout in two coordinates, by strips situated on either side of the chamber. Typical strip pitch is of the order of $1 \mathrm{~cm}$.

The RPCs are relatively cheap, light and easy to handle. They are extensively used for large muon detectors in collider and neutrino experiments. However, they need HV of approximately $10 \mathrm{kV}$ and a careful control of the gasmixture supply, which might constitute relevant inconveniences if employed in a volcanic environment.

\subsection{Plastic scintillators}

The early muon radiographies of volcanoes (Nagamine et al., 1995; Tanaka et al., 2003, 2005) made use of approximately $10 \mathrm{~cm}$ wide plastic-scintillator strips, covering a total area of the order of $1 \mathrm{~m}^{2}$. Each strip was read by a photomultiplier (PMT) through a light guide. The neutrino oscillation experiment OPERA at the Gran Sasso National Laboratory (http://www.cern.ch/opera) employed on a large scale plastic-scintillator strips read out by WaveLength Shifting optical fibres and multianode PMTs for charged-particle tracking.

Figure 7 shows the scintillator strips of the OPERA experiment and their readout system. Each strip is $2.6 \mathrm{~cm}$ wide and about $7 \mathrm{~m}$ long. A groove in the strip allocates a wavelength shifting optical fibre. The fibre conveys the optical signal to a multianode PMT, which reads the signal of 64 strips. Modules of 64 strips and their readout are assembled together, to form planes with approximate size of $7 \times 7 \mathrm{~m}^{2}$. Two planes of strips, with horizontal and vertical orientation, are grouped together to predict the event location in the emulsion detector upstream of them. In total, the plastic scintillator strip system covers an area of 2,900 $\mathrm{m}^{2}$, very large compared to our needs.

A plastic-scintillator detector requires a minimal maintenance, has low power consumption and is relatively insensitive to ambient conditions. The spatial resolution can be optimised by the strip geometrical profile and width. Plastic scintillator strips constitute the most suitable detector option.

\section{Conclusions}

The pioneering studies conducted in Japan demonstrated that radiography by cosmic-ray muons is a powerful new technique to investigate the inner structure of the edifice of volcanoes. In perspective, muon radiography is likely to have a considerable expansion also to other applications.

Given the morphology of the Mt. Vesuvius-Mt. Somma complex, the investigation of the internal structure of the edifice of Mt. Vesuvius at depths larger than in previous volcano radiographies is a real challenge. However, it is motivated by a high social and scientific interest. In order to cope with the strong reduction of the muon flux due to the traversal of the volcano's edifice, a muon-detector active area of tens of square metres is needed, much larger than in previous investigations. The detector design will take advantage of the experience gained from the construction of large area muon detectors for particle-physics experiments. A large detector active area will allow reducing the measurement time and eventually result in muon radiography to be used for almost real-time monitoring purposes. The detector will make use of plastic scintillators and aim at an angular resolution of the order of $15 \mathrm{mrad}$, so far achieved only with nuclear emulsions.

The deployment of a large-area detector at Mt. Vesuvius by an international collaboration is also intended to pave the way to subsequent application to other volcanoes around the world. Of special scientific interest will be the radiography of Strombolian volcanoes.

\section{References}

Acocella, V. et al., Understanding shallow magma emplacement at volcanoes: orthogonal feeder dikes during the 2002-2003 Stromboli eruption, Geophys. Res. Lett., 33, L17310, 2006.

Adair, R. K. and H. Kasha, Cosmic-ray muons, in Muon Physics, edited by V. W. Hughes and C. S. Wu, vol. 1, p. 323, Academic Press, 1976.

Alvarez, L. W. et al., Search for hidden chambers in the pyramids, Science, 167, 832, 1970.

Amsler, C. et al., The Review of Particle Physics, Phys. Lett., B667, 1, 2008.

Auger, E. et al., Seismic evidence of an extended magmatic sill under Mt. Vesuvius, Science, 294, 1510, 2001.

Chouet, B. et al., Shallow-conduit dynamics at Stromboli Volcano, Italy, imaged from waveform inversions, Geological Society, London, Special Publications, 307, 57-84, doi:10.1144/SP307.5, 2008.

Corcella, G. et al., HERWIG 6.5, JHEP, 0101, 010, [hepph/0011363]; hep$\mathrm{ph} / 0210213,2001$. 
George, E. P., Cosmic rays measure overburden of tunnel, Commonwealth Engineer, July 1, 455, 1955.

Gonzalez-Garcia, M. C. et al., Radiography of Earth's core and mantle with atmospheric neutrinos, Phys. Rev. Lett., 100, 061802, 2008.

Miele, G. et al., The Aperture for UHE tau neutrinos of the Auger fluorescence detector using a digital elevation map, Phys. Lett. B, 634, 137, 2006.

Nagamine, K. et al., Method of probing inner structure of geophysical substance with the horizontal cosmic ray muons and possible application to volcanic eruption prediction, Nucl. Instr. Meth., A356, 585, 1995.

Plinius Caecilius Secundus C. (Pliny the Younger), Letters to C. Tacitus VI.16 and VI.20 (about 104).

Tanaka, H. K. M. and I. Yokoyama, Muon radiography and deformation analysis of the lava dome formed by the 1944 eruption of Usu, Hokkaido-Contact between high-energy physics and volcano physics—, Proc. Jpn. Acad., Ser. B, 84, 2008.

Tanaka, H. K. M. et al., Development of a two-fold segmented detection system for near horizontally cosmic-ray muons to probe the internal structure of a volcano, Nucl. Instr. Meth., A507, 657, 2003.

Tanaka, H. K. M. et al., Radiographic measurements of the internal structure of Mt. West Iwate with near-horizontal cosmic-ray muons and future developments, Nucl. Instr. Meth., A555, 164, 2005.

Tanaka, H. K. M. et al., Development of an emulsion imaging system for cosmic-ray muon radiography to explore the internal structure of a volcano, Mt. Asama, Nucl. Instr. Meth., A575, 489, 2007a.

Tanaka, H. K. M. et al., High resolution imaging in the inhomogeneous crust with cosmic-ray muon radiography: The density structure below the volcanic crater floor of Mt. Asama, Japan, Earth Planet. Sci. Lett., 263, 104, 2007b.

Tanaka, H. K. M. et al., Imaging the conduit size of the dome with cosmicray muons: The structure beneath Showa-Shinzan Lava Dome, Geophys. Res. Lett., 34, 389, 2007c.

Tanaka, H. K. M. et al., Radiographic imaging below a volcanic crater floor with cosmic-ray muons, Am. J. Sci., 308, 843, 2008.

Zollo, A. et al., Seismic evidence for a low-velocity zone in the upper crust beneath Mount Vesuvius, Science, 274, 592, 1996.

Zollo, A. et al., Bayesian estimation of 2-D P-velocity models from active seismic arrival time data: imaging of the shallow structure of Mt. Vesuvius, Geophys. J. Int., 151, 566, 2002.

S. Buontempo, L. D’Auria, G. De Lellis, G. Festa, P. Gasparini, G. Iacobucci (e-mail: giuseppe.iacobucci@na.infn.it), A. Marotta, M. Martini, G. Miele, P. Migliozzi, O. Pisanti, P. Strolin, M. Vassallo, and A. Zollo 